\title{
"gnparser": a powerful parser for scientific names based on Parsing Expression Grammar
}

\author{
Dmitry Y. Mozzherin ${ }^{1 * \dagger}$ (D), Alexander A. Myltsev ${ }^{2 \dagger}$ and David J. Patterson ${ }^{3}$
}

\begin{abstract}
Background: Scientific names in biology act as universal links. They allow us to cross-reference information about organisms globally. However variations in spelling of scientific names greatly diminish their ability to interconnect data. Such variations may include abbreviations, annotations, misspellings, etc. Authorship is a part of a scientific name and may also differ significantly. To match all possible variations of a name we need to divide them into their elements and classify each element according to its role. We refer to this as 'parsing' the name. Parsing categorizes name's elements into those that are stable and those that are prone to change. Names are matched first by combining them according to their stable elements. Matches are then refined by examining their varying elements. This two stage process dramatically improves the number and quality of matches. It is especially useful for the automatic data exchange within the context of "Big Data" in biology.

Results: We introduce Global Names Parser (gnparser). It is a Java tool written in Scala language (a language for Java Virtual Machine) to parse scientific names. It is based on a Parsing Expression Grammar. The parser can be applied to scientific names of any complexity. It assigns a semantic meaning (such as genus name, species epithet, rank, year of publication, authorship, annotations, etc.) to all elements of a name. It is able to work with nested structures as in the names of hybrids. gnparser performs with $\approx 99 \%$ accuracy and processes 30 million name-strings/hour per CPU thread. The gnparser library is compatible with Scala, Java, R, Jython, and JRuby. The parser can be used as a command line application, as a socket server, a web-app or as a RESTful HTTP-service. It is released under an Open source MIT license.

Conclusions: Global Names Parser (gnparser) is a fast, high precision tool for biodiversity informaticians and biologists working with large numbers of scientific names. It can replace expensive and error-prone manual parsing and standardization of scientific names in many situations, and can quickly enhance the interoperability of distributed biological information.
\end{abstract}

Keywords: Biodiversity, Biodiversity informatics, Scientific name, Parser, Semantic parser, Names-based cyberinfrastructure, Scala, Parsing Expression Grammar

\section{Background}

\section{Conventions}

Throughout the paper we use the terms "name", "scientific name", and "name-string" in particular ways. "Name" refers to one or several words that act as a label for a taxon. A "scientific name" is a name formed in compliance with a nomenclatural code (Code) or, if beyond the scope of the Codes, is consistent with the expectations of a Code.

\footnotetext{
*Correspondence: mozzheri@illinois.edu

${ }^{\dagger}$ Equal contributors

${ }^{1}$ University of Illinois, Illinois Natural History Survey, Species File Group, 1816

South Oak St., Champaign, IL, 61820, USA

Full list of author information is available at the end of the article
}

The term "name-string" is the sequence of characters (letters, numbers, punctuation, spaces, symbols) that forms the name. A name can be expressed in the form of many name-strings (for example, see Fig. 1). There are about two and a half million currently accepted names for extinct and extant species. There are approximately ten million of legitimately formed scientific names and hundreds of millions of possible name-strings for them. We use the term "elements" for the components of a name-string. Traditionally, in biological literature, scientific names for genera and taxa below genus are presented in italics. In this paper, where we wish to emphasize examples of name-strings, we use bold font. 


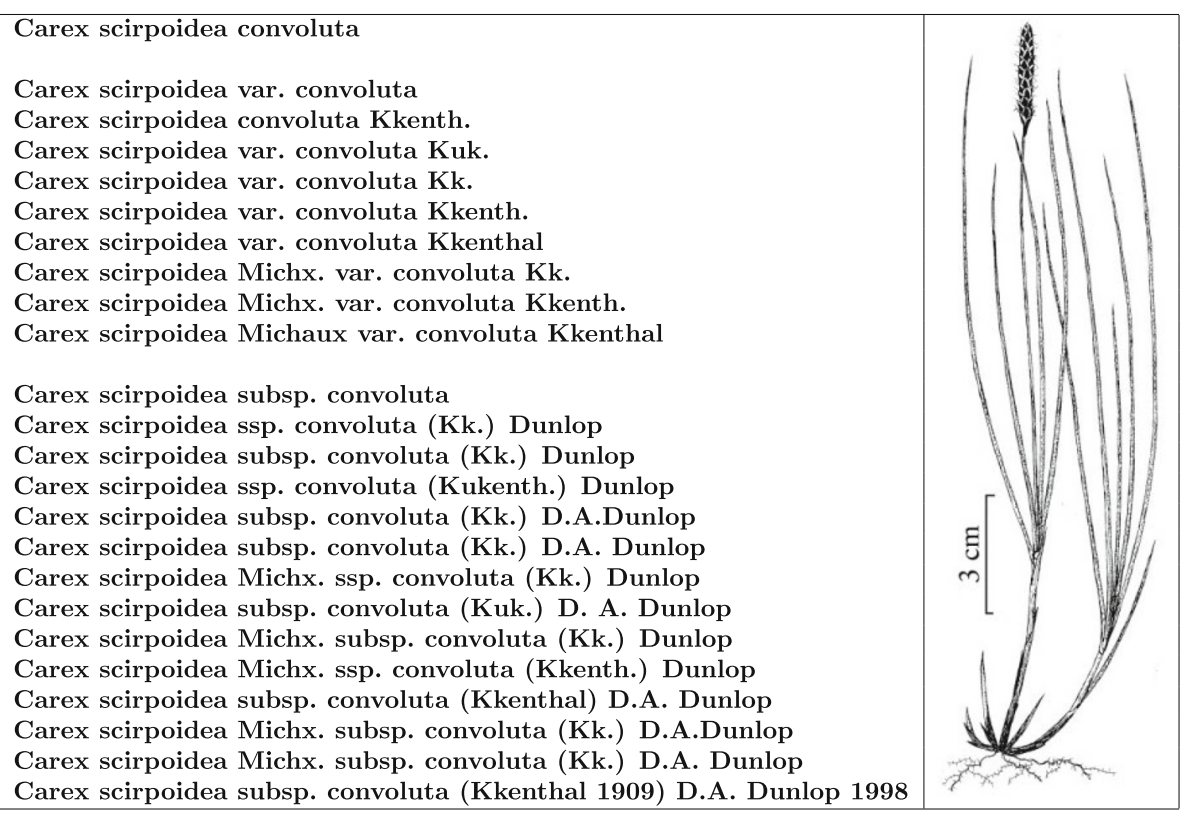

Fig. 1 Some legitimate versions of the scientific name for the 'Northern Bulrush' or 'Singlespike Sedge'. The genus (Carex), species (scirpoidea), and subspecies (convoluta) may be annotated (var., subsp., and ssp.) or include or omit the name of the original authority for the infraspecies (Kükenthal), or for the species (Michaux), or for the current infraspecific combination (Dunlop). The name of the authority is sometimes abbreviated, sometimes differently spelled, and may be with or without initials and dates. This list is not complete. Image courtesy of [42]

\section{Introduction}

Biology is entering a "Big Data" age, where global and fast access to all knowledge is envisaged. Progress towards this vision is still limited in scope. One impediment, especially for the long tail of smaller sources (of which some are not yet digital), is the absence of devices to inter-connect distributed data. The names of organisms are invaluable in "Big Data" biology because they can be treated as metadata and as such can be used to discover, index, organize, and interconnect distributed information about species and other taxa [1]. The use of names for informatics purposes is not straightforward because, for example, there may be many legitimate spellings for a name (Fig. 1). A cyberinfrastructure that uses names to manage information about organisms must determine which name-strings are variant forms of the same scientific name.

Figure 1 presents some of the different legitimate variants of a scientific name in order to make the point that there is not a single correct way to spell scientific names. Because of these variations, fewer than $15 \%$ of the names in comparisons of large biological databases could be matched based on exact spellings of name-strings [2]. In order to improve this simple metric for interoperability, we need to identify variants of the same name. We refer to the process of addressing variant spellings (there being other causes of different names for the same taxon) as "lexical reconciliation". Lexical reconciliation involves linking the alternative spelling variants for the same taxon into a "lexical group". Most biologists do this intuitively - they recognize that the name-strings in Fig. 1 refer to the same taxon. They do so by "parsing" the name-strings into elements (genus name, species name, authors, ranks etc.) and mentally discarding less significant elements such as annotations and authorship. It then becomes clear all of name-strings are formed around the Latin elements Carex scirpoidea convoluta. We refer to the form of the scientific name without authority or annotations as the "canonical form". Further analysis of the name-strings reveals two different lexical groups (separated in Fig. 1 by a line break) for, probably, one taxonomic concept:

- Carex scirpoidea var. convoluta description by Kükenthal

- Carex scirpoidea subsp. convoluta rank determination by Dunlop.

In the past, the need to parse scientific names to form normalized names has mostly been achieved manually. A person familiar with rules of botanical nomenclature would be able to analyse the 24 name-strings in this example with relative ease, but not thousands or millions of name-strings - especially if they include scientific names to which more than one nomenclatural code may be applied. The manual splitting of names into 
even only two parts - the latinized elements of taxon names that make up the canonical form and the authorship - is slow and therefore expensive. To scale this exercise up requires an algorithmic solution, a scientific name parser!

The strategy of the algorithmic approach is to identify which combinations of the most atomic parts of a name-string (i.e. the UTF-8 encoded characters) represent words (such as genus name, species name, authors, annotations) or dates. An early algorithmic approach to parsing scientific names was with "regular language" implemented as regular expression [3]. A regular expression is a sequence of characters that describes a search pattern [4]. For example, a regular expression "[A-Z] [a-z]\{2\}" recognizes a word that starts from a capital letter followed by two small letters (e.g. "Zoo"). Scientific names almost universally follow patterns that are influenced by the Codes of Nomenclature: such as the use of spaces to separate words, capitalization of generic names and authors, or the inclusion of four digit dates between the middle of the 18th century and the present. This makes most names amenable to parsing by regular expressions. Current examples of scientific name parsers based on regular expressions are GBIF's name-parser [5], and YASMEEN [6].

While regular expression is a powerful approach to string parsing, it has limitations. It cannot elegantly deal with name-strings where an authorship element is present in the middle of the name (for example Carex scirpoidea Michx. subsp. convoluta (Kük.) D.A.Dunlop). Indeed, regular expressions are not well suited to any targets with recursive (nested) elements [7], such as hybrid formulae (e.g. Brassica oleracea L. subsp. capitata (L.) DC. convar. fruticosa (Metzg.) Alef. $\times$ B. oleracea L. subsp. capitata (L.) var. costata DC.). Name parsing built on regular expressions is impractical for complex name-strings.

Another limitation with most regular expression software tools is that they are "black boxes" that allow developers very limited interaction with the parsing process. They do not reveal much information about the parsing context and developers cannot call a procedure during a parsing event. As a result, complex regular expression-based parsers are difficult to implement and maintain, and functions such as error recovery, detailed warnings, descriptions of errors are missing.

We wanted to deal with scientific names across a very broad range of complexity and to give more flexibility than can be achieved with a regular expression approach. We believe that a scientific name parser should satisfy the following requirements.

1. High Quality. A parser should be able to break names into their semantic elements to the same standards that can be achieved by a trained nomenclaturalist or better. This will give users confidence in the automated process and allow them to set aside tedious and expensive manual parsing.

2. Global Scope. A parser should be able to parse all types of scientific names, inclusive of the most complex name-strings such as hybrid formulae, multi-infraspecific names, names with multilevel authorships and so on. No name-strings should be left unparsed, otherwise biological information attached to them may remain undiscoverable.

3. Parsing Completeness. All information included in a name-string is important, not just the canonical form of the scientific name. Authorship, year, rank information allow us to distinguish homonyms, similar names, synonyms, spelling mistakes, or chresonyms. Access to such information improves the performance of subsequent reconciliation (the mapping of all alternative name-strings for the same taxon against each other).

4. Speed. Users, especially large-scale aggregators of biodiversity data, are more satisfied with speedy processing of data as it allows them to move forward to more purposeful value-adding tasks. Speed reduces the purchasing/operating costs of the hardware used for production parsing.

5. Accessibility. To be available to the widest possible audience, a parser should be released as a stand-alone program, have good documentation, be able to work as a library, to function as a command line tool, as a tool within a graphical interface, to run as a socket or as RESTful services.

These requirements became our design goals. Based on our experience with prototype systems, we chose to use Parsing Expression Grammar and Scala language.

\section{Adoption of Parsing Expression Grammar}

Parsing Expression Grammar (PEG) [8] have been introduced for parsing strings. PEG allows developers to define the rules ("grammar") that describe the general structure of target strings. Such rules can be used to deconstruct scientific names. The rules are built from the ground up, starting from the simplest - such as a combination of "characters" separated by "spaces". That 'rule' identifies most "words". Digits and other characters make dates identifiable. Further rules can be applied, such as a "genus" rule can describe a part of a polynomial name-string in which the first word begins with combination of a "capital_character" followed by several "lower_case_characters" that fall within a relatively small spectrum of allowed characters; "authorship" would consist of one or more capitalized words and followed perhaps by a "year". Within some instances of authorship, authors may be grouped to form "author-teams". PEG rules are designed to be recursive. They can be 
expanded to deal with increasingly complex name-strings, or address errors such as absent or extra spaces, or OCR errors. Each rule can have programmatic logic attached, making the PEG approach very flexible. We believe that PEG suits our goals better than regular expressions for the following reasons:

- PEG is better suited than regular expressions for strings with a recursive structure;

- the syntax of scientific names is formal enough to be closer to an algebraic structure rather than to a natural language. Inconsistencies and ambiguities in scientific name-strings are relatively rare because they usually comply with the requirements and conventions of nomenclatural codes;

- scientific name-strings are short enough to avoid problems with computational complexity and memory consumption;

- programming a parser with PEG can describe parsing rules in a domain-specific language;

- domain-specific languages offer great flexibility for logic within the rules, for example to report errors in name-strings.

The Global Names project created a specialized parsing library biodiversity in 2008 [9]. It was written in Ruby and based on PEG. It uses the TreeTop Ruby library [10] as the underlying PEG implementation.

The PEG approach allowed us to deal with complex scientific names gracefully. It gave us flexibility to incorporate edge cases and to detect common mistakes during the parsing process. The biodiversity library has enjoyed considerable popularity. At the time of writing, it had been downloaded more than 150,000 times [11], it is used by many taxon name resolution projects (e.g. Encyclopedia of Life [12], Canadian Register of Marine Species (CARMS) [13], the iPlant TNRS [14], and World Registry of Marine Species (WoRMS) [15]. According to statistics compiled by BioRuby, biodiversity, at the time of writing, has been the most popular bio-library in the Ruby language [16].

We were pleased with PEG approach for parsing scientific names, but regard the biodiversity parser library as a working prototype. It has allowed us to make further improvements and deliver a better, faster productiongrade parser.

\section{Other approaches}

There is a growing number of algorithms and tools in machine learning and natural language processing that aim to recognize parts of texts. They include statistical parsing [17], context-free grammars [18], fuzzy contextfree grammars [19], and named entity recognition [20]. Unsupervised deep learning $[21,22]$ increases the quality of entity recognition without extensive curation and programming efforts by people. We chose not to use these approaches for the following reasons.

- The limited scope of a parser. A parser of scientific names very rarely needs to work with name-strings of more than 15 words.

- There is no need for recognition. A scientific namestring parser is usually applied to preexisting lists of scientific names. There is no requirement to recognize scientific names in larger bodies of text. Other scientific name recognition and discovery tools are available.

- Formal grammar. Scientific names are formed in compliance with well-defined and formal codes of nomenclature. They have predictable structures making the requirements for a scientific name-string parser to be more similar to parsers of programming languages than to tools designed to work with natural languages.

- Scale and throughput. We created the parser to serve the needs of biodiversity aggregators. A core design requirement was to develop a lightweight library for inputs of millions of scientific name-strings per second, and to be processed locally.

- Stand-alone approach. We did not wish the parser to rely on local or remote previously known information of genera, species, author names, or other scientific names. gnparser relies instead on morphological features of scientific name-strings.

- Determinism. Biologists know that there is only a single correct parsed version of a scientific name. A scientific names parser must produce a single "correct" result for each input string. A parser should provide meta information on every part of the string.

\section{Adoption of Scala}

The pre-existing biodiversity package is not speedy and cannot scale because it uses Ruby as its programming language. Ruby is one of the best languages for rapid prototyping, but it is an interpreted dynamic language with, originally, a single-threaded runtime during execution. This makes it slow and inappropriate for "Big Data" tasks. We concluded that we needed a replacement language environment with the following properties:

- a mature technology;

- multithreaded, with high performance and scalability;

- an active support community with an Open source friendly culture;

- a wide range of libraries: utilities, web frameworks, etc.;

- a powerful development environment with IDEs, testing frameworks, debuggers, profilers and the like;

- mature libraries for search and cluster computations;

- interoperable with languages popular in scientific community ( $R$, Python, Matlab); 
- natural support of domain specific languages embedded in the hosted language.

While many of the properties are true for Ruby, other properties, such as high performance, scalability and interoperability, are not. To meet all requirements, and exploiting what we had learned from biodiversity, we rewrote the code using Scala (a Java virtual machine programming language [23]), and the Open source parboiled2 library [24] which we improved [25]. The parboiled2 library implements PEG in Scala. An alternative to parboiled2 is the Scala combinators library [26]. We did not use it because it is slow and has memory consumption problems.

The functional programming features of Scala allowed us to build a domain specific language that describes the grammar's rules to parse scientific names. This produces a Parsing Expression Grammar with considerably more flexibility than external lexers such as Bison or Yacc. As this domain specific language is within parboiled2, it can take advantage of the Macro capacity of Scala [27] to optimize the compilation of the code and the subsequent running of the program. As a result, the software performs with high efficiency. The resulting gnparser library is faster, more scalable and more flexible than its predecessor.

We limited this version to work with scientific names that comply with the botanical, zoological, and prokaryotic codes of nomenclature, but not with names of viruses because they are formed in different ways $[2,28]$ and need a different PEG. We intend to add this later.

\section{Implementation}

The gnparser project is entirely written in Scala. It supports two major Scala versions: 2.10.6+ and 2.11.x. The code is organized into four modules:

1. "parser" is the core module used by all other modules. It parses scientific names from the most atomic components of a name-string to semantically-defined terms. It includes the parsing grammar, an abstract syntax tree (AST) composed of the elements of scientific names, warning and error facilities. When the parsing is complete and semantic elements of name-strings have been assigned to AST nodes, the elements can be recombined and formatted to meet further needs. For example:

- normalizer converts input name-strings into a consistent style;

- canonizer creates canonical forms of the latinized elements of names;

- JSON renderer, the parsing result is converted to JSON [29] to allow developers to work with the output using other languages. The output (Fig. 2, also see Results and discussion) has the following information: 'details' contains the
JSON-representation of a parsed scientific name; 'quality_warnings' describes potential problems if names are not well-formed; 'quality' depicts a quality level of the parsed name; and 'positions' maps the positions of every element in a parsed name to the semantic meaning of the element. Full and formal explanation of all parser fields is given as a JSON schema and can be found online [30] [also see Additional file 1].

2. The "spark-python" module contains facilities to use "gnparser" with Apache Spark scripts written in Python. Apache Spark is a highly distributive and scalable development environment for processing massive sets of data. Spark is written in Scala, but can also be used with Python, R and Java languages. Spark programs written in Java and Scala are able to run "parser" in a distributed fashion natively.

3. The "examples" module contains examples to assist developers in adding "parser" functionality into other popular programming languages such as Java, Scala, Jython, JRuby, and R.

4. The "runner" module contains the code that allows users to run "parser" from a command line as a standalone tool or to run it as a TCP/IP socket or HTTP web server. It depends on the "parser" module. The core part is the launch script "gnparse" (for Linux/Mac and Windows) that creates a JVM instance and runs "parser" on multiple threads against the input provided via a socket or file. This module also contains a web application and a RESTful interface to offer simpler ways to access "parser". "web" achieves interactions with "parser" via HTTP protocol. It works both with simple web (HTML) and REST API interfaces. Figure 2 illustrates a parsing example using the web-interface. Socket and REST services use Akka framework which makes them highly concurrent and scalable.

"parser" and "examples" can run in JVM 1.6+. "runner" requires JVM 1.8+. Documentation is available in a README file [see Additional file 2].

\section{Parsing rules}

gnparser v0.3.1 contains 76 PEG rules. In turn, these rules make use of more elementary rules provided by the parboiled2 library. The rules are domain-specific based on hours of conversations with leading taxonomists, study of nomenclatural codes, and feedback of the users.

As an example, the yearNumber rule is given below. It detects the year in which a name was published. Rule[Year] is a type of the returning value of the rule. Using domain-specific language and elementary rules of parboiled 2 we capture the start and the end positions of a year substring (lines \#1 and \#2). This matches a substring that represents a year in scientific name-strings. A 


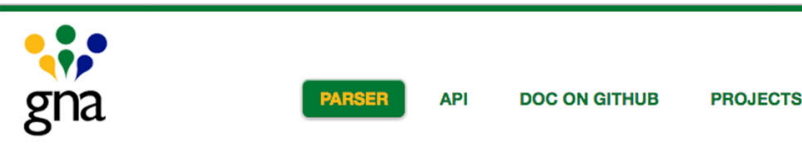

\section{Global Names Parser \\ Scientific Names in Detail}

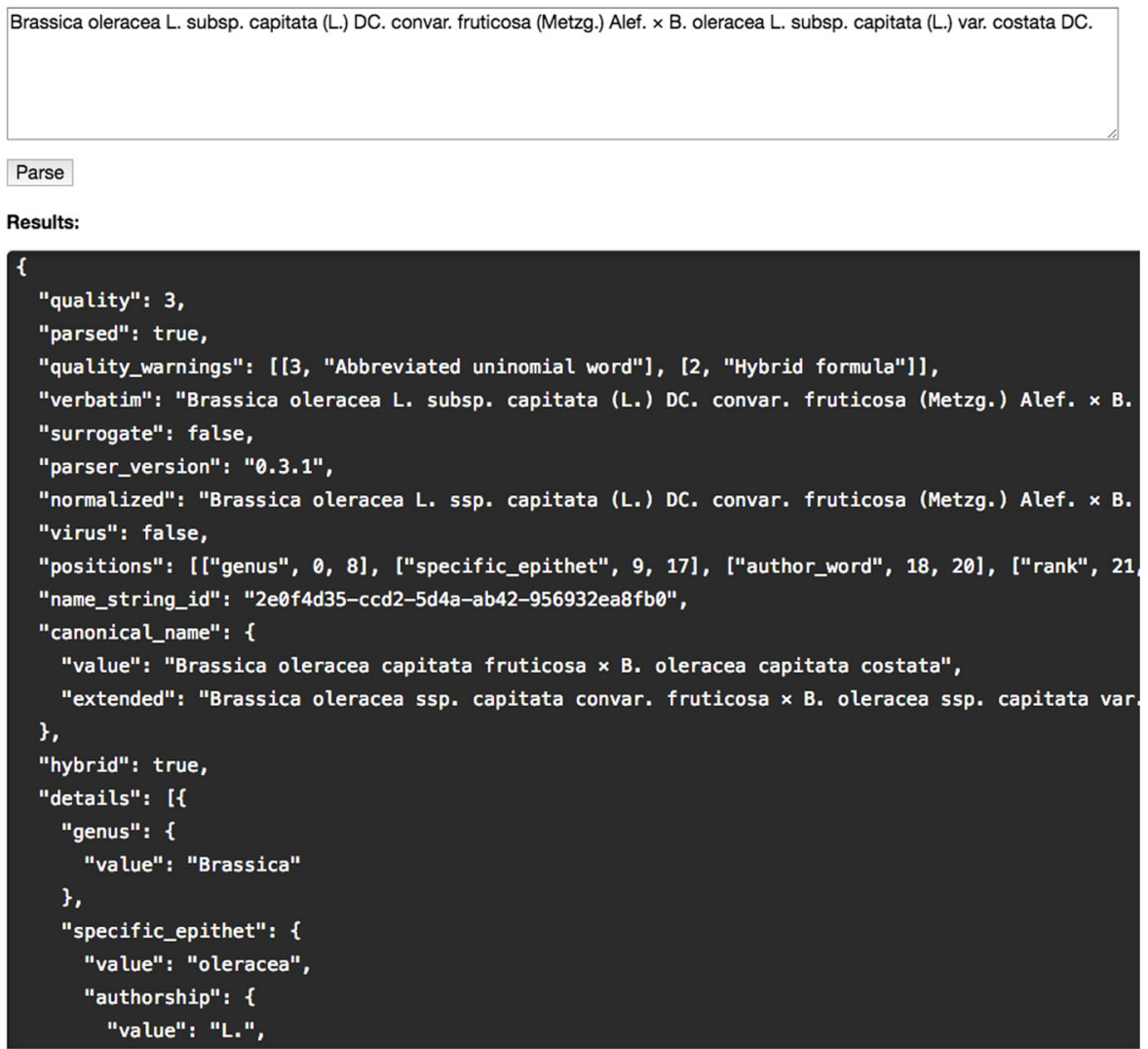

Fig. 2 Web Graphical User Interface [43]. In this example a user entered a name-string of a hybrid name consisted of 21 elements. The "Results" section contains detailed parsed output using compact JSON format

publication year is usually a number between 1753 [31] and the present. A year substring might have one or two digits substituted with question marks if the exact year of a publication is unknown. The capture is then passed as a parameter to a parser action (line \#3). Parser action, a Scala function, might produce warnings or a class instance of defined type (Rule[Year]).

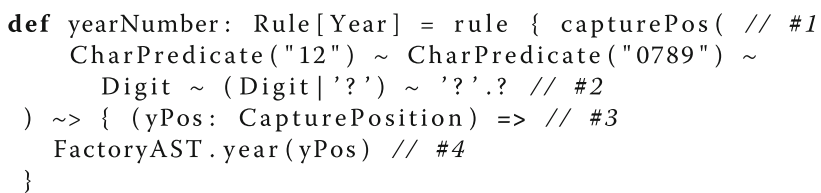

We then assemble more complex inter-dependent rules (lines \#5 to \#10), and finally combine all of them into the rule year on line \#11 that consists of prioritized alternatives of all previously defined rules.

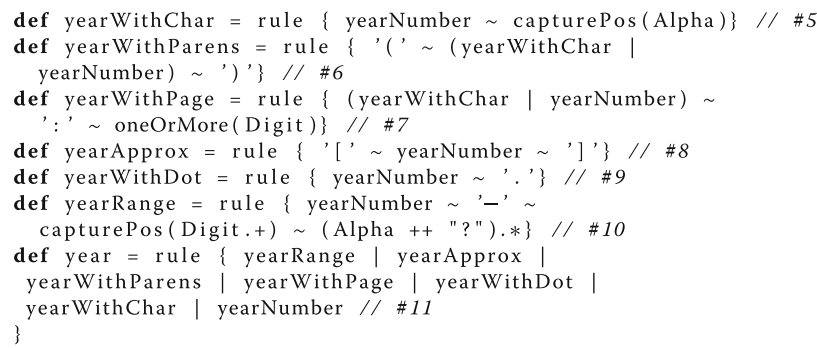

This enables the incorporation of the year rule into all cases where it might be needed. For example on line \#12 we indicate that year must be present in the matcher for the authorsYear rule. 
def authorsYear: RuleNodeMeta[AuthorsGroup] = rule \{

authorsGroup softSpace (', $\sim$ softSpace).? year $\sim\{\mid / \# 12$

(aM: NodeMeta [AuthorsGroup], yM: NodeMeta[Year]) $\Rightarrow$

val $\mathrm{a} 1=$ for $\{\mathrm{a}<-\mathrm{aM} ; \mathrm{y}<-\mathrm{yM}\}$ yield a.copy $($ year $=y \cdot$ some $)$

a1. changeWarningsRef ((aM. node, a1. node $))$

\section{Installation}

"gnparser" is available for launch in three bundles.

- A parser artifact is provided via the Maven central repository of Java code [32]. Physically it is a relatively small jar file without embedded external dependencies. The artifact can be accessed in custom projects by a build system such as Maven, Gradle, or SBT. The build system identifies and provides access to all dependent jars.

- A Zip-archived "fat jar" is located at the project's GitHub repository. The jar contains the compiled files of gnparser along with all necessary dependencies to launch it within JVM. The archive is also bundled with a launch script (for Windows, OS X and Linux) that can run a command line interface to gnparser.

- The project's Docker container image is located at Docker Hub [33]. Docker provides an additional layer of abstraction and automation of operating-systemlevel virtualization on Linux. It can be thought of as a lightweight virtualization technology within a Linux OS host. When it is setup properly, everything starting from JVM and ending with Scala and SBT - can be run with simple commands that will, for example, pull the gnparser's Docker image from the DockerHub, and run the socket or web server on an appropriate port.

\section{Testing methods}

Data for our tests were sets of 1000 and 100,000 name-strings randomly chosen from 24 million unique name-strings of the Global Names Index (GNI) [34]. The name-strings in GNI are collected from a large variety of biodiversity data sources and are pre-identified as scientific names. While GNI contains some incorrectly classified strings, it is the largest compilation of name-strings representing scientific names. It is not biased towards any particular taxon or particular variant of name, and so the extracted datasets are believed to represent naturally occurring data quite well. The datasets are randomly chosen and are therefore mixtures of well-formed names, lexical variants of names, names with formatting and spelling mistakes, and name-strings that were misrepresented as names. Name-strings in the sets are independent of each other. An evaluation dataset with 1000 names is included as Additional file 3.

We compared the performance of gnparser with two other projects: biodiversity parser [9, 35] (also developed by Global Names team), and the GBIF name-parser [5]. The following versions were used: gnparser v. 0.2.0, GBIF name-parser v. 0.1.0, biodiversity v. 3.4.1. To make comparisons, we calculated Precision, Recall and Accuracy (as described below) using a dataset consisting of 1000 name-strings. We also tested the YASMEEN parser from iMarine [6]. With our dataset, YASMEEN generated many more mistakes than other parsers (Precision 0.534, Recall 1.0, F1 0.6962), and was unable to finish a full dataset without crashing. We excluded it from further tests.

To estimate the quality of the parsers, we relied on their performance in representing canonical forms and terminal authorships. A canonical form represents the latinized elements of taxon names, while the terminal authorship refers to the author of the lowest subtaxon found in the scientific name. For example, with Oriastrum lycopodioides Wedd. var. glabriusculum Reiche, the canonical form is Oriastrum lycopodioides glabriusculum and the terminal authorship is Reiche, not Wedd.

When both the canonical form and the terminal authorship were determined correctly we marked the result as true positive $\left(N_{t p}\right)$. If one or both of them were determined incorrectly, the result was marked as a false positive $\left(N_{f p}\right)$. Name-strings correctly discarded from parsing were marked as true negatives $\left(N_{t n}\right)$. False negatives $\left(N_{f n}\right)$ were name-strings which should have been parsed, but were not. The results of the tests are summarized in Table 1:

Accuracy - the proportion of all results that were correct. It is calculated as:

$$
\text { Accuracy }=\frac{N_{t p}+N_{t n}}{N_{t p}+N_{t n}+N_{f p}+N_{f n}}
$$

Precision - the proportion of name-strings parsed correctly compared to all detected name-strings. It is calculated as:

$$
\text { Precision }=\frac{N_{t p}}{N_{t p}+N_{f p}}
$$

Recall - the proportion of correctly detected namestrings relative to all parseable name-strings and is calculated as:

Table 1 Precision/Recall for parsers applied to 1000 name-strings

\begin{tabular}{llll}
\hline & gnparser & gbif-parser & Biodiversity \\
\hline True positive & 978 & 955 & 971 \\
True negative & 13 & 12 & 13 \\
False positive & 9 & 32 & 16 \\
False positive & 0 & 1 & 0 \\
Precision & 0.989 & 0.968 & 0.984 \\
Recall & 1.0 & 0.999 & 1.0 \\
F1 & 0.994 & 0.983 & 0.992 \\
Accuracy & 0.989 & 0.967 & 0.984 \\
\hline
\end{tabular}




$$
\text { Recall }=\frac{N_{t p}}{N_{t p}+N_{f n}}
$$

The $F 1$ - measure is a balanced harmonic mean (where Precision and Recall have the same weight). When Precision and Recall differ, F1 - measure allows results to be compared. It is calculated as

$$
F 1=\frac{2 \times \text { Precision } \times \text { Recall }}{\text { Precision }+ \text { Recall }}
$$

Some names in the dataset were not well-formed. If a human could extract the canonical form and the terminal authorship from them, we included them in our assessment. Examples of such name-strings are "Hieracium nobile subsp. perclusum (Arv. -Touv. ) O. Bolòs \& Vigo" (the problem for the parser here is an introduced space within an author's name), "Campylium gollanii $C$. M?ller ex Vohra 1970 [1972]" (with a miscoded UTF8 symbol and an additional year in square brackets), “Myosorex muricauda (Miller, 1900)." (with a period after the authorship).

Parsers analyze the structure of name-strings, but they cannot determine if a string is a "real" name. For example, in the case of a name-string that has the same form as a subspecies such as "Example name Word var. something Capitalized Words, 1900". In such a case, the identification of a canonical form as "Example name something" and terminal authorship as "Capitalized Words, 1900" would be considered a true positive. Clearly, it will be important for name-management services to distinguish between name-strings of scientific names, names of viruses, surrogate names, and nonnames. To find out how well parsers distinguished strings which are not scientific names, we calculated Accuracy for discarded/non-parsed strings. If the parser worked well, non-parsed strings would include only names of viruses and terms that do not comply with the codes of zoological, prokaryotic, and botanical nomenclature.

We processed 100,000 name-strings with each parser. Each parser discarded close to 1,000 name-strings as nonparseable. Accuracy, in this case, provided the percentage of correctly discarded names out of all discarded by the parser names. We do not know Recall, as it was not reasonable to manually determine this for 100,000 names. To get a sense of names which should be discarded but were parsed instead, we analysed intersections and differences of the results between the three parsers as shown in Table 2.

To establish the throughput of parsing we used a computer with an Intel i7-4930K CPU (6 cores, 12 threads, at $3.4 \mathrm{GHz}$ ), 64GB of memory, and 250GB Samsung 840 EVO SSD, running Ubuntu version 14.04. Throughput was determined by processing 1,000,000 random namestrings from Global Names database.
Table 2 Accuracy of non-parseable names detection out of 100,000 name-strings

\begin{tabular}{llll}
\hline & gnparser & gbif-parser & Biodiversity \\
\hline True discarded & 1131 & 1082 & 1161 \\
Correctly discarded & 1129 & 940 & 1152 \\
Incorrectly discarded & 2 & 142 & 9 \\
Accuracy & 0.998 & 0.869 & 0.992 \\
\hline
\end{tabular}

To study the effects of parallel execution on throughput we used the ParallelParser class from biodiversity parser. We used 'gnparse file -simple' (a command line-based script set to return simplified output) for gnparser. For GBIF name-parser, we created a thin wrapper with multithreaded capabilities [36]. The following versions had been used for throughput benchmarks: gnparser v. 0.3.1, GBIF name-parser v. 0.1.0, biodiversity v. 3.4.1.

\section{Results and discussion}

We discuss and compare gnparser, GBIF name-parser and biodiversity parser in the context of our requirements for quality, global scope, parsing completeness, speed, and accessibility.

\section{High quality parsing}

Quality is the most important of the 5 requirements. GBIF name-parser uses regular expressions approach, while gnparser and biodiversity parsers use the PEG approach. Results for quality measurements are shown in Tables 1 and 2. We include the 1,000 tested names as Additional file 3.

If test data contain a large proportion of true negatives $\left(N_{t n}\right)$ Accuracy will not be a good measure as it favors algorithms that distinguish negative results rather than finding positive ones. We manually checked our test datasets and established that $\approx 1 \%$ were not scientific names. Given that true negatives are rare, they will have very limited influence on Accuracy. Recall for all parsers was high, hence false negatives are not important.

Accuracy is probably the best measure for our tests. All 3 parsers performed very well, with Accuracy values higher than 95\%. Both gnparser and biodiversity parser approached the $99 \%$ mark which we regard as the metric for production quality. Most of the false positives came from name-strings with mistakes. For example, out of 11 false positives (below) that gnparser found in the 1000 name-string test data set, only 2 (the first 2 ) were wellformed names.

\section{Eucalyptus subser. Regulares Brooker Jacquemontia spiciflora (Choisy) Hall. fil. Acanthocephala declivis variety guianensis Osborn, 1904 \\ Atysa (?) frontalis}


Bumetopia (bumetopia) quadripunctata Breuning, 1950 Cyclotella kã $1 / 4$ tzingiana Thwaites

Elaphidion (romaleum) tæniatum Leconte, 1873

Hieracium nobile subsp. perclusum (Arv. -Touv. ) $O$. Bolòs \& Vigo

Leptomitus vitreus (Roth) Agardh?

Myosorex muricauda (Miller, 1900).

Papillaria amblyacis $(\mathrm{M}<\mathbf{8 1}>\mathbf{l l}$.Hal.) A.Jaeger

We do expect a parser to deal with names that are not well-formed. That means overcoming problems such as aberrant characters which might arise from Unicode character miscodings, inappropriate annotations, or other mistakes. To alert users, gnparser generates a warning when it identifies a problem in a name-string. The other parsers do not have this feature.

When parsers reach $\approx 80 \%$ Accuracy, they hit a "long tail" of problems where each particular type of a problem is rare. Every new manual check of additional test sets of 1,000-10,000 name-strings reveals new issues. Examples of these challenges are given elsewhere [2]. For all three parsers, developers have to perform the meticulous task of adding new rules to address each rare case. That is, parsers need to be subject to continuous improvement. The problems found during preparation of this paper are being addressed in the next version of gnparser. As the parsing rules improve, we believe that gnparser can reach $>99.5 \%$ Accuracy without diminishing Recall.

As we incorporate new rules to increase Recall, we have to consider the risks of reducing Precision by introducing new false positives. For example, the GBIF name-parser allows the genus element of a name-string to start with a lowercase character. As a result the name-strings below were parsed as if they were scientific names, while the other parsers ignored them:

acid mine drainage metagenome agricultural soil bacterium CRS5639T18-1 agricultural soil bacterium $\mathrm{SC}-\mathrm{I}-8$ algal symbiont of Cladonia variegata MN075 alpha proteobacterium AP-24 anaerobic bacterium ANA No.5 anoxygenic photosynthetic bacterium G16 archaeon enrichment culture clone AOM-SR-A23 bacterium endosymbiont of Plateumaris fulvipes bacterium enrichment culture DGGE band 61_3_FG_L barley rhizosphere bacterium JJ-220 bovine rumen bacterium niuO17

Strategies like these may increase Recall with certain low-quality datasets, but they decrease Precision. Many "dirty" datasets contain recurring problems. As an example, DRYAD contains many name-strings in which elements of scientific names are concatenated with an interpolated character such as ', (e.g. "Homo_sapiens" and "Pinoyscincus_jagori_grandis") [2]. For them, our solution was to include a "preparser" script which "normalizes" known problems that are inherent within particular datasets and then apply a high quality parser to the result.

Our testing also revealed differences between regular expressions and PEG approaches. Both can achieve high quality results with canonical forms of scientific names, but the regular expressions are less suitable for more complex name-strings. The recursive or nested nature of some scientific names can cause problems which become insurmountable for regular expressions.

\section{Global scope}

If we want to connect biological data using scientific names, no name-strings should be missed or rejected, no matter how complex they are. During our testing we found that Accuracy of GBIF's name-parser was depressed because, in part,the parser did not recognize hybrid formulae and infrasubspecific names with more then one infraspecific epithet. This case underscores the limitations of the regular expression approach. As examples, the following were not parsed by the GBIF name-parser:

Erigeron peregrinus ssp.callianthemus var. eucallianthemus (a name-string with two infraspecificx epithets)

Polyporus varius var. nummularius f. undulatus (Pilát) Domanski, Orlos \& Skirg. (two infraspecific epithets)

Salvelinus fontinalis $x$ Salmo gairdneri (hybrid formula)

Echinocereus fasciculatus var. bonkerae $\times E$. fasciculatus var. fasciculatus (hybrid formula)

The PEG approach supports nested parsing rules to create progressively more complex rules that manage such cases. The capacity to address recursion allows gnparser to handle the full spectrum of scientific names that we have presented to it.

\section{Parsing Completeness}

The extraction of canonical forms from name-strings representing scientific names is the most beneficial and widely used parsing goal. Sometimes, however, this may not be sufficient because the canonical form does not always distinguish a name completely.

In the example in Fig. 1 Carex scirpoidea convoluta is a canonical form for Carex scirpoidea var. convoluta Kükenthal and Carex scirpoidea ssp. convoluta (Kük.) Dunlop. The first non-parsed name-string refers to the variety convoluta of Carex scirpoidea that had been described by Kükenthal. The second 
captures Dunlop's reclassification of convoluta as a subspecies. We are not able to distinguish between these two different names without knowing the rank and/or the corresponding authorship. Furthermore, it is useful to see in the second example that (Kük.) was the original author and Dunlop was the author of the new combination. Also, canonical forms do not distinguish between homonyms. The heather, Pieris japonica (Thunb.) D. Don ex G. Don and the butterfly, Pieris japonica Shirôzu, 1952 have the same canonical form Pieris japonica.

After matching by canonical form, rank, authors, and "types" of authorship allow us to distinguish name-strings with similar or identical canonical elements. The namestring Carex scirpoidea Michx. var. convoluta Kükenth. adds the information that the species Carex scirpoidea was described by Michx but is not evident in the examples in the paragraph above.

Another area in which parsers with limited abilities can give misleading results is with negated names [2]. In these cases, the name-string includes some annotation or marks to indicate that the information associated with the name does NOT refer to the taxon with the scientific name that is included. Examples include Gambierodiscus aff toxicus or Russula xerampelina-like sp.

All components of a name may be important and need to be parsed and categorized. With gnparser, we describe the meaning of every element in the parsed name-string and present the results in JSON format. Parsing of Carex scirpoidea Michx. subsp. convoluta (Kük.) D.A. Dunlop gives the following JSON output
The output includes the semantic meaning of all parsed elements in a name-string, indicates if the namestring was parsed successfully, if it is a virus name, a hybrid, or a surrogate. Surrogates are name-strings that are alternatives to names (such as acronyms) and they may or may not include part of a scientific or colloquial name (e.g. Coleoptera sp. BOLD:AAV0432). The output also includes a statement of the position of each element in the name-string. Last, but not least, the JSON output contains UUID version 5 calculated from the verbatim name-string. This UUID is guaranteed to be the same for the same namestring, promoting its use to globally connect information and annotations.

The output usually covers every semantic element in the name-string. The fields in the output illustrated above have the following meanings.

name_string_id: UUID v5 identifier;

parsed: whether a name-string was successfully parsed (true/false);

quality: how well-formed a name-string is (range from 1 to 3,1 is the best);

parser_version: version of a parser used;

verbatim: name-string as was submitted to gnparser;

normalized: name-string modified by the parser to give a normalized style;

canonical_name: a special form of normalization that includes only the scientific elements of the name, this form is contained within most name-strings relating to scientific names;

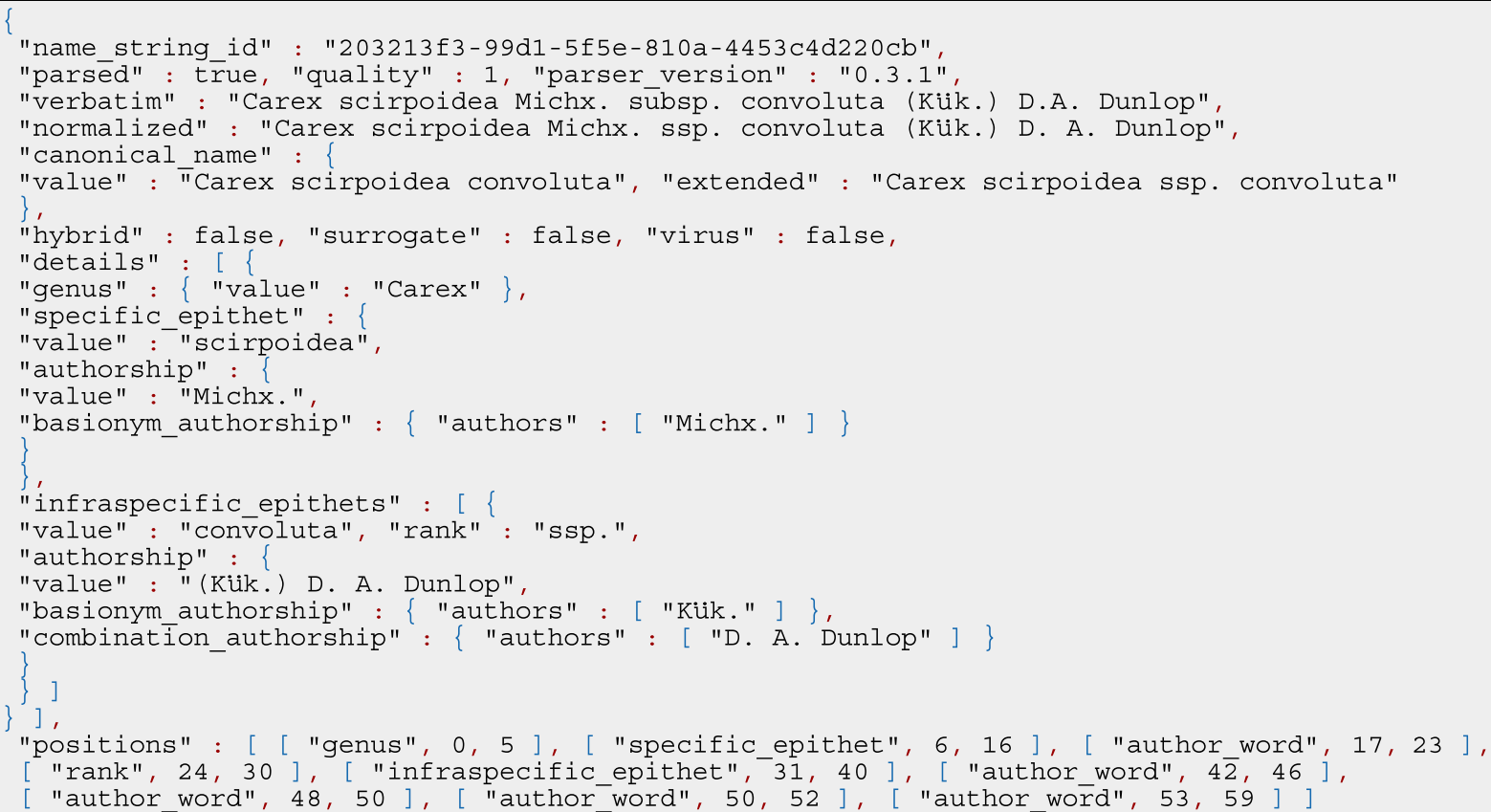


hybrid: whether the name-string refers to a hybrid (true/false);

surrogate: whether a name-string is a surrogate name (true/false);

details: describes the semantic elements within the name-string inclusive of the following;

genus: reports the genus part of the name (in this case Carex);

specific epithet: reports the species epithet (scirpoidea); authorship: reports the authorship of the combination (Michx.);

basionym authorship: reports the authorship of the basionym (Michx.)

infraspecific epithets: reports the infraspecies name if present (convoluta) with rank (ssp.)

authorship: reports the authors of the infraspecies name ((Kük.) D. A. Dunlop)

basionym authorship: reports the author of the basionym of infraspecies name element ([“Kük."]);

combination authorship: reports the author of the infraspecies name combination (D. A. Dunlop); and

positions: identifies each name element and where it starts and ends.

The complete list of fields for the gnparser's output exists as a JSON Schema file [30] [see Additional file 1].

\section{Parsing speed}

In the areas of performance discussed above, there is little difference between biodiversity parser and gnparser. There is, however, a dramatic difference in their parsing speed and ability to scale. Parsing tasks that took 20 hours with earlier biodiversity parsers can now be completed in a few minutes on a multithreaded computer. Parsing is a key to other services such as name-reconciliation and subsequent resolution. Improvements to the speed of the parser will increase user satisfaction elsewhere.

Results on the speed performance are given in Fig. 3. The performance depends on the number of CPU threads used. On 1 thread gnparser was 7 times faster than biodiversity, 10 times faster on 4 threads, and 14 times faster on 12 threads.

gnparser displays functionality not presented in the GBIF name-parser as described in previous sections. In spite of this additional functionality gnparser outperformed other tested parsers.

\section{Accessibility}

By 'accessibility' we refer to the ability of the software code to be used by a wide audience. For Open source projects, accessibility is very important. If more people use a software, the more cost-effective is its development.

Parsing scientific names is essential for organizing biodiversity data. Many biodiversity database environments and projects include a parsing algorithm. Examples are

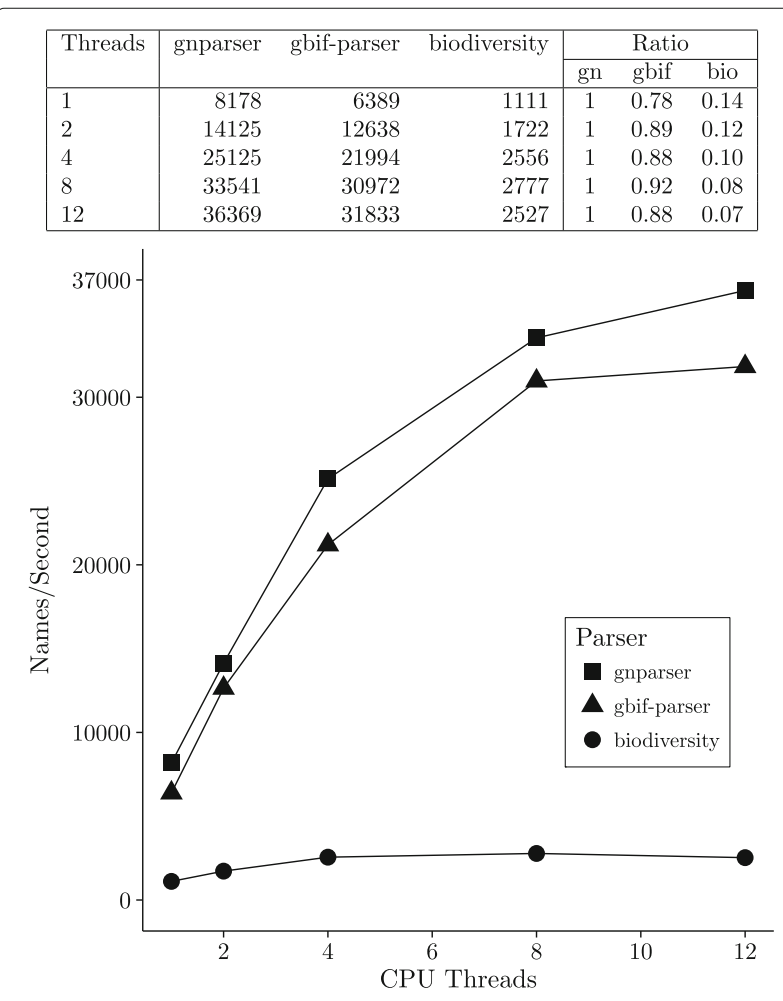

Fig. 3 Names parsed per second by GN, GBIF and Biodiversity parsers (running on 1-12 parallel threads)

uBio [37], the Botanical Society of Britain and Ireland [38], FAT [39], NetiNeti [40], and Taxonome [41]. A modular approach offers an option of re-use and avoids replication of effort. biodiversity was the first biodiversity parser to be released as a stand-alone package that could be used as a module - as it was with the iPlant project [35]. The same approach has now been adopted with the GBIF name-parser [5], YASMEEN [6], and gnparser.

We designed gnparser with accessibility in mind from the outset. Scala language allows the use of gnparser as a library in Scala, Java, Jython, JRuby and a variety of other languages based on Java Virtual Machine it can also be used natively in $\mathrm{R}$ and Python via JVM-binding libraries. Apache Spark, a "Big Data" framework, is also supported. The following example illustrates how a client written in Jython can access the gnparser functionality.

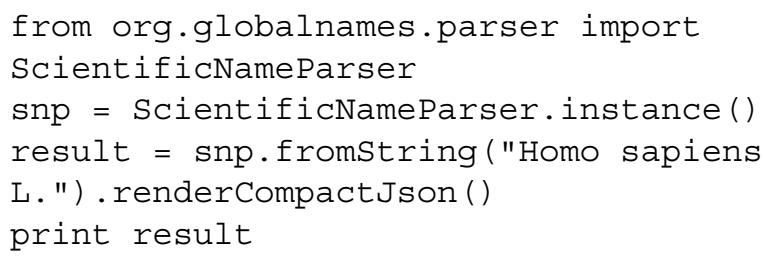

If programmers want to use gnparser in some JVMincompatible language they can connect to the parser via a socket server interface. There is also a command line tool, 
a web interface, and a RESTful API. In 2016, Encyclopedia of Life started to parse name-strings using gnparser socket server.

We pay close attention to documentation, trying to keep it detailed, clear, and up to date. We have an extensive test suite [see Additional file 4] that describes the parser's behavior and contains examples of gnparser functionality and output format.

This commitment to accessibility creates a larger potential audience for the parser, and will help many researchers and programmers deal with the problems that arise from variant forms of scientific names.

\section{Conclusions}

The performance of the scientific names parsers is summarised in Table 3. The two PEG-based parsers - biodiversity and gnparser are similar. They are based on the same algorithmic approach and follow similar design goals. While we had the option of modifying the rules for biodiversity to improve Accuracy, we preferred to create a new tool from scratch to overcome limitations in speed, scalability and accessibility. We needed to address speed at Global Names because existing software took too long to parse or reparse 24 million name-strings. gnparser can be used natively by larger variety of programming languages than biodiversity, because JVM-based languages and tools are so widely used. Our first goal for gnparser was complete coverage of the biodiversity's test suite. We continue to improve gnparser while biodiversity entered maintenance mode. That explains a slight difference in Accuracy by these two parsers.

gbif-parser is a high quality product. However, its regular expressions-based algorithm limits its usability. The recursive nature of some scientific names creates significant obstacles for intrinsically non-recursive algorithms such as regular expressions. Coverage of multiinfraspecific names and hybrids, both with recursive patterns, is prohibitively expensive for such an approach.

Table 3 Summary comparison of Scientific Name Parsers

\begin{tabular}{llll}
\hline & gnparser & gbif-parser & Biodiversity \\
\hline Accuracy & $98.9 \%$ & $96.7 \%$ & $98.4 \%$ \\
Hybrid formulas support & Yes & No & Yes \\
Infrasubspecies support & Yes & No & Yes \\
Throughput (names/s/thread) & 8178 & 6389 & 1111 \\
Parsing details & Complete & Partial & Complete \\
Library for the samelanguages & Yes & Yes & Yes \\
Library for other languages & Yes & Yes & No \\
Command line tool & Yes & No & Yes \\
Socket server & Yes & No & Yes \\
Web interface & Yes & Yes & Yes \\
RESTful service & Yes & Yes & Yes \\
\hline
\end{tabular}

In conclusion, this paper describes gnparser, a powerful tool for working with biodiversity information. It transforms names of taxa into their semantic elements. This allows standardization of names by, for example, representing them as canonical forms. This step dramatically improves name matching within and among data sources, and this increases the amount of data on a single taxon that can be integrated. Parsing can be used to improve the discovery of names in sources, and creating a common taxonomic index to multiple sources. Parsing allows users to extract, compare and analyse metadata within the name-strings, and allowing comparisons of the efforts of individuals or to map trends over time. The gnparser tool is released under MIT Open source license, contains command line executable, socket, web, and REST services, and is optimized for use as a library in languages like Scala, Java, R, Jython, JRuby.

\section{Availability and requirements \\ Project Name: gnparser}

Project home page: https://github.com/GlobalNames Architecture/gnparser

Operating System: Any platform able to run JVM 1.8

Programming Language: Scala

License: The MIT License

Other requirements: docker (optional)

Any restrictions to use by non-academic: no restriction

The data supporting the conclusions of this article are available in the repository https://github.com/ GlobalNamesArchitecture/gnparser-paper under the data directory.

\section{Additional files}

Additional file 1: Includes a full and formal explanation of all parser fields as a JSON schema. (JSON $9 \mathrm{~kb}$ )

Additional file 2: README.rst file that is converted to HTML format. It is also available at project home page [44]. (ZIP 7 kb)

Additional file 3: 1,000 name-strings randomly selected from $\mathrm{GNI}$ and used to determine Accuracy, Precision and Recall data (Table 1). (TXT $32 \mathrm{~kb}$ )

Additional file 4: Extensive test suite that describes the parser's behavior. It is also a source of examples of parser functionality and output format. Test suite consists of a pipe delimited input (scientific name) and parsed output in JSON format. (TXT 253 kb)

\section{Abbreviations}

AAM: Alexander A. Myltsev; API: Application program interface; AST: Abstract Syntax Tree; BHL: Biodiversity heritage library; DJP: David J. Patterson; DYM: Dmitry Y. Mozzherin; GBIF: Global Biodiversity information facility; GNA: Global names architecture; GNI: Global names index; JSON: JavaScript object notation; JVM: Java virtual machine; PEG: Parsing expression grammar; REST: Representational state transfer

\section{Acknowledgements}

The authors thank David Mark Welch (Josephine Bay Paul Center, Marine Biological Laboratory) for the leadership at the beginning of the gnparser project. The authors also thank administrators of the Species File Group for the much needed support during the transfer of the GNA grant from the Marine Biological Laboratory to University of Illinois. 


\section{Funding}

This work is supported by the National Science Foundation (NSF DBI-1356347). The Species File Group of the University of Illinois provided an additional funding. The funding bodies had no role in the study design, data collection and analysis, decision to publish, or preparation of the manuscript.

\section{Authors' contributions}

DYM and AAM designed gnparser. DYM created requirements, test suite and the original version of gnparser. AAM optimized gnparser for speed, refactored it into three internal subprojects. DYM set Docker containers and Kubernetes scripts. DYM and AAM wrote online documentation and JSON schema to formalize output. DJP corrected parser's results, calibrated quality output and errors output. DYM and AAM drafted manuscript and DJP edited its final version. All authors read and approved the final manuscript.

\section{Competing interests}

The authors declare that they have no competing interests.

\section{Consent for publication}

Not applicable.

\section{Ethics approval and consent to participate}

Not applicable.

\section{Declarations}

All authors have gone through the manuscript and contents of this article have not been published elsewhere.

\section{Publisher's Note}

Springer Nature remains neutral with regard to jurisdictional claims in published maps and institutional affiliations.

\section{Author details}

${ }^{1}$ University of Illinois, Illinois Natural History Survey, Species File Group, 1816 South Oak St., Champaign, IL, 61820, USA. ${ }^{2}$ IP Myltsev, Kaslinskaya St., Chelyabinsk, 454084, Russia. ${ }^{3}$ University of Sydney, Sydney, Australia.

\section{Received: 21 October 2016 Accepted: 28 April 2017}

Published online: 26 May 2017

\section{References}

1. Patterson DJ, Cooper J, Kirk PM, Pyle RL, Remsen DP. Names are key to the big new biology. Trends Ecol Evol. 2010;25(12):686-91. doi:10.1016/j.tree.2010.09.004.

2. Patterson D, Mozzherin D, Shorthouse D, Thessen A. Challenges with using names to link digital biodiversity information. Biodiversity Data J. 2016:4:8080. doi:10.3897/BDJ.4.e8080.

3. Leary PR, Remsen DP, Norton CN, Patterson DJ, Sarkar IN. uBioRSS: Tracking taxonomic literature using RSS. Bioinformatics. 2007;23(11): 1434-6. doi:10.1093/bioinformatics/btm109.

4. Aho AV, Ullman JD. Foundations of Computer Science vol. 2. USA: Computer Science Press New York; 1992.

5. GBIF name-parser. https://github.com/gbif/name-parser/releases/tag/ name-parser-2.10. Accessed 18 Apr 2017.

6. Vanden Berghe E, Coro G, Bailly N, Fiorellato F, Aldemita C, Ellenbroek A, Pagano P. Retrieving taxa names from large biodiversity data collections using a flexible matching workflow. Ecol Inform. 2015;28:29-41. doi:10.1016/j.ecoinf.2015.05.004.

7. Yu S. Handbook of formal languages, regular languages. New York: Springer Verlag; 1997.

8. Ford B. Parsing Expression Grammars: A Recognition-Based Syntactic Foundation. In: Proceedings of the 31st ACM SIGPLAN-SIGACT, ACM, New York, 2004. Symposium on Principles of Programming Languages; 2004. p. 111-22.

9. GlobalNamesArchitecture/biodiversity: Scientific Name Parser. https:// github.com/GlobalNamesArchitecture/biodiversity. Accessed 18 Apr 2017.

10. Treetop. https://github.com/cjheath/treetop. Accessed 18 Apr 2017.

11. RubyGems - biodiversity search. https://rubygems.org/search?query= biodiversity. Accessed 18 Apr 2017.

12. Encyclopedia of Life. http://eol.org/. Accessed 18 Apr 2017.
13. Canadian Register of Marine Species. http://www.marinespecies.org/ carms/. Accessed 18 Apr 2017.

14. iPlant Taxonomic Name Resolution Service. http://tnrs.iplantcollaborative. org/. Accessed 18 Apr 2017.

15. WoRMS - World Register of Marine Species. http://www.marinespecies. org/. Accessed 18 Apr 2017.

16. Ruby Libraries for Biology. http://biogems.info/. Accessed 18 Apr 2017.

17. Charniak E. Statistical Language Learning. USA: MIT Press; 1996.

18. Aho AV, Ullman JD. The Theory of Parsing, Translation, and Compiling. Upper Saddle River: Prentice-Hall, Inc.; 1972.

19. Asveld PRJ. A fuzzy approach to erroneous inputs in context-free language recognition. In: Proceedings of the Fourth International Workshop on Parsing Technologies IWPT'95. Prague, Czech Republic: Institute of Formal and Applied Linguistics, Charles University; 1995. p. 14-25.

20. Nadeau D, Sekine $S$. A survey of named entity recognition and classification. Lingvisticae Investigationes. 2007;30(1):3-26. doi:10.1075/li.30.1.03nad

21. Mikolov T, Sutskever I, Chen K, Corrado GS, Dean J. Distributed representations of words and phrases and their compositionality. In: Advances in Neural Information Processing Systems 26. USA: Curran Associates, Inc.; 2013. p. 3111-9.

22. Schmidhuber J. Deep learning in neural networks: An overview. Neural Netw. 2015;61:85-117.

23. Odersky M, Altherr P, Cremet V, Emir B, Maneth S, Micheloud S, Mihaylov N, Schinz M, Stenman E, Zenger M. An overview of the Scala programming language. Technical report. 2004.

24. Myltsev A, Doenitz M. parboiled2: a macro-based approach for effective generators of parsing expressions grammars in Scala 2017. in preparation. http://myltsev.com/papers/parboiled2.pdf. Accessed 10 May 2017.

25. GlobalNamesArchitecture/parboiled2: A macro-based PEG parser generator for Scala 2.10+ doi:10.5281/zenodo.50340. https://github.com/ GlobalNamesArchitecture/parboiled2. Accessed 18 Apr 2017

26. Moors A, Piessens F, Odersky M. Parser combinators in Scala. Department of Computer Science, KU Leuven, Leuven, Belgium. 2008.

27. Burmako E. Scala macros: Let our powers combinel: On how rich syntax and static types work with metaprogramming. In: Proceedings of the 4th Workshop on Scala SCALA '13. New York: ACM; 2013. p. 3-1310. doi:10.1145/2489837.2489840

28. King AMQ, Adams MJ, Carstens EB, Lefkowitz EJE. Virus Taxonomy: Classification and Nomenclature of Viruses: Ninth Report of the International Committee on Taxonomy of Viruses. Amsterdam: Elsevier Academic Press; 2012, pp. 1-1338.

29. Bray T. The JavaScript object notation (JSON) data interchange format. Google Inc. Online. 2014

30. JSON schema for gnparser output. http://globalnames.org/schemas/ gnparser.json. Accessed 18 Apr 2017.

31. Linne CV. Plantarum: Exhibentes Plantas Rite Cognitas Ad Genera Relatas Cum Differentiis Specificis, Nominibus Trivialibus, Synonymis Selectis, Locis Natalibus Secundum; Holmiae, Impensis Laurentii Salvii, Stockholm, 1753, p. 583.

32. Maven Central: Global Names Artifacts. https://search.maven.org/\# search|gal1/globalnames. Accessed 18 Apr 2017.

33. Global Names Parser Docker Image. https://hub.docker.com/r/gnames/ gnparser/. Accessed 18 Apr 2017.

34. Global Names Index. http://gni.globalnames.org. Accessed 18 Apr 2017.

35. Boyle B, Hopkins N, Lu Z, Raygoza Garay JA, Mozzherin D, Rees T, Matasci N, Narro ML, Piel WH, McKay SJ, Lowry S, Freeland C, Peet RK, Enquist BJ. The taxonomic name resolution service: an online tool for automated standardization of plant names,. BMC Bioinformatics. 2013;14(1):16. doi:10.1186/1471-2105-14-16.

36. gbifparser: v0.1.0 2015. doi:10.5281/zenodo.34848. http://dx.doi.org/10. 5281/zenodo.34848. Accessed 18 Apr 2017

37. uBio Name Parser. http://www.ubio.org/tools/explode.php. Accessed 18 Apr 2017.

38. Botanical Society of Britain and Ireland Taxon Name Parser. http://bsbidb. org.uk/taxonnameparser.php. Accessed 18 Apr 2017.

39. Sautter G, Böhm K, Agosti D. A combining approach to Find All Taxon names ( FAT) in legacy biosystematics literature. Biodivers Inform. 2006;3: 46-58. doi:10.2307/1216144.

40. Akella LM, Norton CN, Miller H. NetiNeti: discovery of scientific names from text using machine learning methods. BMC Bioinformatics. 2012;13(1):211. doi:10.1186/1471-2105-13-211. 
41. Kluyver TA, Osborne CP. Taxonome: a software package for linking biological species data. Ecol Evol. 2013;3(5):1262-5. doi:10.1002/ece3.529.

42. Flora of North America Editorial Committee E. Flora of North America. Vol. 23, Magnoliophyta: Commelinidae (in Part): Cyperaceae. New York and Oxford: Oxford University Press; 2002, p. 551.

43. Global Names Parser Web App. http://parser.globalnames.org. Accessed 18 Apr 2017.

44. GlobalNamesArchitecture/gnparser: Split scientific names to meaningful elements with meta information. https://github.com/ GlobalNamesArchitecture/gnparser. Accessed 18 Apr 2017.

\section{Submit your next manuscript to BioMed Central} and we will help you at every step:

- We accept pre-submission inquiries

- Our selector tool helps you to find the most relevant journal

- We provide round the clock customer support

- Convenient online submission

- Thorough peer review

- Inclusion in PubMed and all major indexing services

- Maximum visibility for your research

Submit your manuscript at www.biomedcentral.com/submit 